\title{
Perspectives on Over Twenty Years of Life Science Research with Atomic Force Microscopy and a Look Toward the Future
}

\author{
B. Ohler* \\ * Veeco Instruments, 112 Robin Hill Road, Santa Barbara, CA 93117
}

The potential for scanning probe microscopy to address topics in the life sciences was recognized even in very early work by scanning tunneling microscope (STM) inventors Binnig and Rohrer. So it should be no surprise that the invention of atomic force microscopy (AFM) by Binnig, Quate, and Gerber in 1986 was soon followed by numerous reports of its application to a wide variety of questions in the life sciences. The capability of AFM to image non-conductive biological samples at high resolution with little or no sample preparation was immediately recognized as a valuable new technique. But two key developments soon thereafter ensured a lasting role for AFM in life sciences. The first was the extension of AFM to operate in liquid environments, which greatly expanded its application to biological systems. The second development was the recognition that AFM has the nearly unique capability to measure force interactions in a range relevant to many biological processes, including those on the single molecule scale.

Since these key developments in the early 1990's, the number of life science publications referencing AFM has grown rapidly worldwide. This growth was supported by an early commercial recognition of the life science market potential, which made the technology available to a larger and diverse group of investigators. These years saw publication of numerous reports of ultra-high resolution imaging of membrane proteins, exploration of a potential role of AFM in DNA sequencing, widespread use in the field of Alzheimer's research, applications to lipid biophysics, a new field of research- "force spectroscopy"- and many other examples, which will be reviewed.

The next milestone in the evolution of AFM life science applications was the combination of AFM with a far more common instrument in biological laboratories, the inverted light microscope. The first commercial example was Digital Instruments "BioScope" AFM in 1994. Such instruments enabled AFM to be more easily used and correlated with results of common light microscopy techniques. However, these early instruments provided only rudimentary physical integration of the two instruments and there was little customization to meet the unique requirements of life science applications.

Today, modern AFMs for life science applications go well beyond mere physical integration with inverted light microscopes. They offer advanced functional integration of the two techniques, including the ability calibrate and register the optical field of view with the "AFM field of view." This allows both automated real-time use of optical images to guide the locations of AFM images and force measurements as well as simple correlation of the different data types in real-time and post-acquisition. There has also been a clear emphasis on improvement of AFMs to meet some of the unique requirements of biological samples, including things like extended scan ranges and stage incubator accessories for long term live cell studies. Examples of research utilizing these latest developments will be presented. 
The future for AFM in the life sciences appears bright. Publication trends suggest that biological applications are one of the most rapidly growing areas of AFM usage. A number of promising technologies have been introduced recently, including modes that make AFM operation simpler and more accessible, modes for fast, quantitative high resolution mapping of material properties and tipsample interactions, and technologies for accelerating AFM imaging rates at speeds sufficient to visualize a broader range of biological processes. Ultimately, all of these exciting applications and technologies remain rooted in the same core strengths of AFM that were recognized very early in its development- the ability to image at high resolution and address single molecules, the ability to operate in liquids under near-physiological conditions, and the ability to measure forces in the picoand nano-Newton range. Perhaps most promising of all is the steady spread of AFM from physics labs and biophysics lab now into molecular and cellular biology labs in both academia and industry. 